\title{
Prolonged survival in adenocarcinoma of unknown primary treated with chemoradiotherapy
}

\author{
Camille Hardy-Abeloos, BS, ${ }^{\mathrm{a}}$ Michael Buckstein, MD, ${ }^{\mathrm{b}}$ Umut Sarpel, MD, ${ }^{\mathrm{c}}$ Monica Prasad \\ Hayes, MD, ${ }^{d}$ and Sofya Pintova, $\mathrm{MD}^{\mathrm{a}}$
}

Departments of a Medicine, Division of Hematology/Oncology, ${ }^{\mathrm{b}}$ Radiation Oncology, ${ }^{\mathrm{c} S u r g e r y}$, Division of Surgical Oncology, and ${ }^{\mathrm{d} G y n e c o l o g y, ~ D i v i s i o n ~ o f ~ G y n e c o l o g i c ~ O n c o l o g y, ~ I c a h n ~ S c h o o l ~ o f ~ M e d i c i n e ~ a t ~ M o u n t ~ S i n a i, ~ N e w ~ Y o r k, ~ N e w ~ Y o r k ~}$

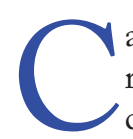
ancer of unknown primary (CUP) represents $3 \%$ to $5 \%$ of all cancer malignancies in the world. ${ }^{1}$ Since 2003 , CUP has been divided into 2 subsets - favorable ( $20 \%$ of the cases) and unfavorable ( $80 \%$ of the cases) - based on histopathologic and clinical manifestations. ${ }^{2}$ The impact of locoregional therapies, such as surgery and radiation, in addition to systemic chemotherapy in adenocarcinomas of unknown primary is not well described in the literature. We report here the case of a patient with adenocarcinoma of unknown primary with lymph-node-only metastases who has remained free of tumor progression for 2 years since completion of systemic multiagent chemotherapy followed by consolidation chemoradiotherapy (CRT).

\section{Case presentation and summary}

A 37-year-old Bengali woman born and raised in Bangladesh, with a history of gallstones diagnosed in 2010, presented to the emergency department at an outside community hospital in New York in the fall of 2014 with right upper-quadrant pain that was more severe after meals during the previous 3 to 6 months. Her past medical history was significant for hypertension, gastroesophageal reflux disease, and kidney stones. She had no past surgical procedures. On family history, both her parents were deceased, and her mother had been diagnosed with hypertension. Her 4 siblings and 2 daughters had no known medical conditions. She did not smoke or drink alcohol and lived with her husband in Queens, New York. On physical exam, her abdomen was soft, nontender, and with normal bowel sounds. An ultrasound on November 10,2014, showed a shadowing stone measuring $1.5 \times 0.9 \mathrm{~cm}$ in the gallbladder fundus. She therefore underwent a cholecystectomy at an outside community hospital in December 2014 and was found to have gallstones and a metastatic adenocarcinoma of a pericholecystic lymph node. No mass was found in the gallbladder. A positronemission and computed-tomographic (PET-CT) scan in January 2015 showed hypermetabolic activity in the porta hepatis. She was scheduled for an upper endoscopy that was cancelled because the results of her beta human chorionic gonadotropin (hCG) test were elevated.

The patient was frustrated by the lack of diagnosis and extensive work-up and decided to travel to Bangladesh for several months. Upon her return in May 2015, the patient underwent dilation and curettage at an outside tertiary care center because of her persistently elevated beta-hCG levels $(>500$ $\mathrm{mIU} / \mathrm{mL}$; reference range for nonpregnant woman, $<5 \mathrm{mIU} / \mathrm{mL}$ ) that found no products of conception and excluded a malignant process. Endoscopy and colonoscopy at that time failed to reveal a primary tumor.

She was then referred to our institution. Her level of beta-hCG remained elevated, and another transvaginal ultrasound was performed but failed to reveal any masses or evidence of pregnancy. Mammogram and a breast ultrasound showed left breast lesions. Biopsy of the breast lesions was performed, and the pathology demonstrated fibrocystic changes.

Because the lymph node was located near the liver, we also measured the patient's alpha fetoprotein (AFP), which is a marker for hepatocellular carcinoma. It was found to be elevated at 1,800.7 $\mathrm{ng} / \mathrm{mL}$ (reference range, $0.0-9.0 \mathrm{ng} / \mathrm{mL}$ ). Elevated

Accepted for publication September 6, 2018. Correspondence: Sofya Pintova, MD; sofya.pintova@mssm.edu. Disclosures: The authors report no disclosures or conflicts of interest. JCSO 2018;16(5):e206-e209. (02018 Frontline Medical Communications. doi: https://doi.org/10.12788/jcso.0424 
serum AFP occurs in pregnancy, nonseminatous germ cell tumors, hepatocellular carcinoma, and other gastrointestinal tumors. The test for AFP has a low sensitivity, so an elevated AFP is not clinically useful in helping identify the origin of the primary tumor. The patient's level of lactate dehydrogenase (LDH), a tumor marker for germ cell tumors, was also elevated at $296 \mathrm{U} / \mathrm{L}$ (reference range, 100-220 U/L). CA 19-9, CA 125, and carcinoembryonic antigen, tumor markers of gas-

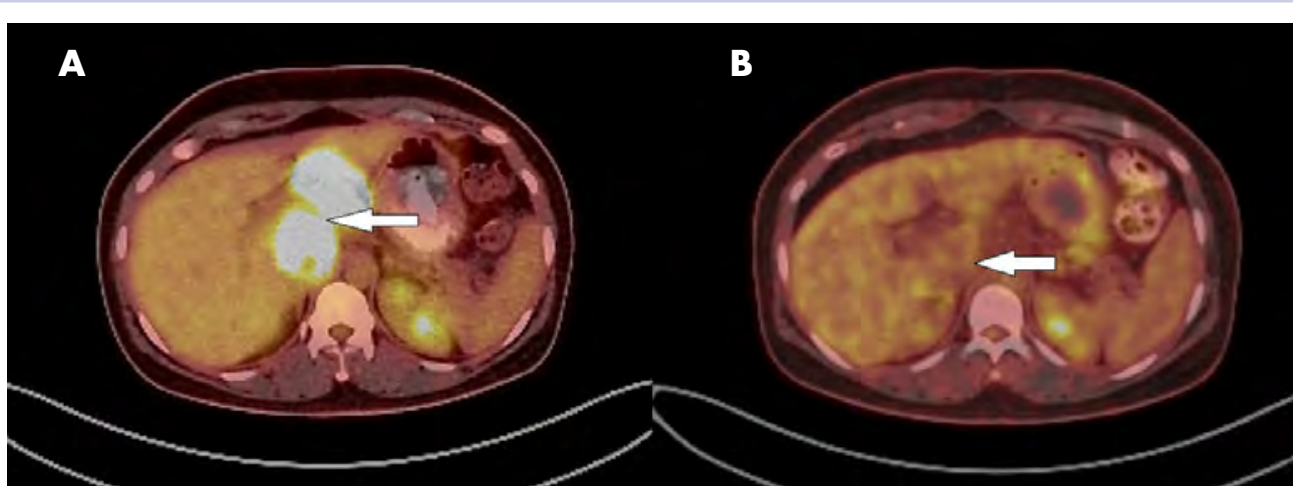

FIGURE I A, Baseline PET-CT scan before any therapy, with porta hepatis lymphadenopathy, with an SUV of 14. B, PET-CT after 4 months of chemotherapy, with porta hepatis lymphadenopathy, with an SUV of 3.5.

PET-CT, positron-emission and computed-tomographic; SUV, standardized uptake value trointestinal carcinomas, did not demonstrate elevated levels at $19.8 \mathrm{U} / \mathrm{mL}$ (reference range, 0.0-35.0 U/mL), $16 \mathrm{U} / \mathrm{mL}$ (reference range, $0-35 \mathrm{U} / \mathrm{mL}$ ), and $0.7 \mathrm{ng} / \mathrm{mL}$ (reference range, $0.0-3.0 \mathrm{ng} / \mathrm{mL}$ ), respectively. No hepatitis serologies were measured at the time of diagnosis.

The results of a PET-CT scan in August 2015 showed a lobulated abdominal mass of $5.7 \times 3.7 \mathrm{~cm}$, consisting of multiple periportal necrotic lymph nodes with a standardized uptake value (SUV) of 14 (Figure 1A) and a $2.0-\mathrm{cm}$ hypermetabolic retroperitoneal lymph node at the aortic bifurcation level with an SUV of 8.6. The SUV is a ratio of activity per unit volume of a region of interest to the activity per unit whole body volume. An SUV of 2.5 or higher is generally considered to be indicative of malignant tissue. We conducted a detailed review of the lymph node pathologic specimen. Immunohistochemical (IHC) studies were positive for CK7, CDX2, and EMA; focally positive for PR and mammaglobin; and negative for CK20, ER, TTF1, and WT-1. Nonspecific staining was seen with BRST2, and there was no staining with GATA3. IHC stain for HER2-NEU was equivocal. Molecular analysis did not detect BRAF, KRAS, NRAS, and PIK3CA mutations, but did find a $C T N N B 1$ mutation. The IHC pattern suggested pancreatobiliary origin of the tumor. ${ }^{3}$

Although serum tumor marker pattern of elevated betahCG, AFP, and LDH can be seen in germ cell tumors, the pathology evaluation did not favor a germ cell tumor. No site of origin was evident on radiographic evaluation, and the patient was diagnosed with CUP. Based on tumor metastatic distribution and the elevated beta-hCG level, ${ }^{4}$ we suspected that an undetected pancreatic primary was possible, and we therefore chose the folinic acid, fluorouracil, irinotecan, oxaliplatin (FOLFIRINOX) chemotherapy regimen for its evidence in prolonging survival in metastatic pancreatic cancer. ${ }^{5}$ At the initiation of treatment, the patient's elevated tumor markers were beta-hCG 953.6 $\mathrm{mIU} / \mathrm{mL}$ (reference for nonpregnant woman,
$<5 \mathrm{mIU} / \mathrm{mL}$ ) and AFP $1,800.7 \mathrm{ng} / \mathrm{mL}$ (reference range, 0.0-9.0 ng/mL). The patient began FOLFIRINOX chemotherapy in August 2015 and after 1 month of treatment, her beta-hCG and AFP levels declined notably to $1.7 \mathrm{mIU} /$ $\mathrm{mL}$ and $11.2 \mathrm{ng} / \mathrm{mL}$, respectively. She completed a total of 8 cycles of FOLFIRINOX in November 2015. After completion of chemotherapy, the PET-CT scan showed a decrease in fluoro-D-glucose (FDG) uptake in the porta hepatis and retroperitoneal lymph nodes (Figure 1B). SUV in the porta hepatis lymph nodes declined from 14 to 3.5. The patient's case was presented to our institution's multidisciplinary tumor board, and the members deemed the risk of possible lymph node dissection surgery would outweigh the benefit. It was recommended that we proceed with radiotherapy to the residual lymph node stations.

During December 2015 through February 2016, the patient underwent a course of consolidative chemoradiation therapy to the intra-abdominal lymph nodes to a dose of 5,400 cGy in 30 fractions, with concurrent capecitabine as radiosensitizer, using intensity-modulated radiation therapy. During both chemotherapy and CRT, the patient experienced nausea, vomiting, fatigue, and anorexia, which were treated with antiemetics. She completed therapy without major complications and recovered completely from the adverse effects.

Five weeks after completion of chemoradiation, a restaging PET-CT scan showed a persistent small FDG uptake in the periportal region (SUV, 4.2). After CRT, tumor markers beta-hCG and AFP declined to less than 1.2 $\mathrm{mIU} / \mathrm{mL}$ and less than $2.0 \mathrm{ng} / \mathrm{mL}$, respectively.

Three and a half years after diagnosis and 2.5 years after completion of the treatment course, the patient remains free of cancer progression without any therapy. Restaging $\mathrm{CT}$ scans of the chest, abdomen, and pelvis every 3 to 6 months continue to show an amorphous soft tissue density in the porta hepatis, which has remained unchanged throughout the last 2 years since chemoradiation 


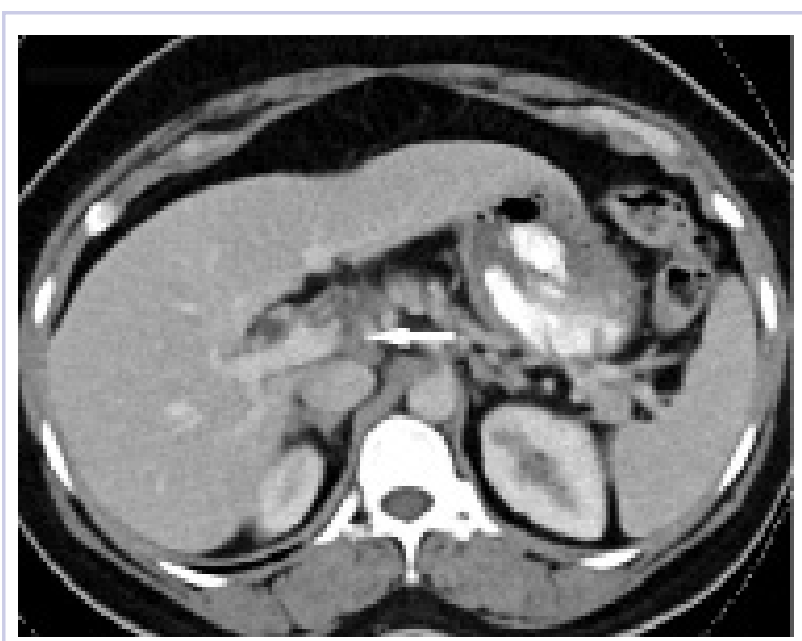

FIGURE 2 Computed-tomographic scan of the patient's chest, abdomen, and pelvis 24 months after chemoradiation therapy showing amorphous soft tissue density in the porta hepatis.

(Figure 2). The levels of the patient's tumor markers AFP and beta-hCG remain normal.

\section{Discussion}

CUP is divided into favorable and unfavorable subsets. ${ }^{1}$ The favorable subset includes women with adenocarcinoma involving axillary lymph nodes, women with papillary adenocarcinoma of peritoneal cavity, and adenocarcinoma with a colon profile. The unfavorable subset includes moderate to poorly differentiated adenocarcinomas (64\%) and undifferentiated tumors (36\%). It involves the liver in $40 \%$ to $50 \%$ of the cases, followed by lymph nodes (35\%), lungs (31\%), bones (28\%), and the brain (15\%)., ${ }^{1,2,6}$ Although data suggest that CUP with lymph-node-only metastases generally fall into an unfavorable prognosis group, our patient's survival and progression-free survival have been especially prolonged. Remarkably, our patient is still alive 44 months after the diagnosis.

The combined platinum-paclitaxel-based regimens are the treatment of choice in this unfavorable subset of CUP, ${ }^{7,8}$ with patients showing $16 \%$ to $38 \%$ response rates and median overall survival times of 6.5 to 13 months. ${ }^{7}$ Platinum-gemcitabine combinations can also be used as an alternative first-line regimen, with an overall response rate of $55 \%$ and a median survival of 8 months. ${ }^{9}$ The addition of the targeted agents bevacizumab and erlotinib to the carboplatin-paclitaxel combination, followed by bevacizumab and erlotinib maintenance, has been shown to yield a median survival of 12.6 months but was not meaningfully superior to historical studies with chemotherapy alone. ${ }^{10}$

We chose the FOLFIRINOX regimen for our patient. Conroy and colleagues reported a notably improved survival of 11.1 months with that combination chemotherapy in patients with metastatic pancreatic cancer compared with 6.8 months with gemcitabine alone. ${ }^{5}$ Given the possible pancreatobiliary site of tumor origin on IHC, the lymph node pattern of spread, and the patient's young age and robust performance status, we felt that this multiagent systemic therapy would offer the best chance of prolonged survival. FOLFIRINOX includes a platinum agent, oxaliplatin, and platinum agents are recommended to be included in chemotherapy combinations for CUP. ${ }^{9,10}$ Although there is no data to suggest the superiority of a triplet regimen over a doublet regimen in a CUP, a triplet chemotherapy regimen may be considered in select cases.

There have been only a few reports showing the effectiveness of radiotherapy in the treatment of adenocarcinomas of unknown primary outside of the head and neck. Kubisch and colleagues have reported a case of a woman with hepatic adenocarcinoma of unknown primary that was treated with chemotherapy and surgery. Upon recurrence, the patient was then treated with selective internal radiation therapy (SIRT). She was still alive 3 years after diagnosis, and there had been no tumor relapse 21 months after SIRT. ${ }^{11}$ Shiota and colleagues have reported a case of a mediastinal lymph node CUP that was treated with docetaxel and cisplatin with concurrent thoracic radiation therapy. ${ }^{12}$ The patient remained free of symptoms without regrowth of the primary site 22 months after disease onset, and exploration of the body with enhanced and PET-CT scan showed no further abnormalities.

Other reports suggest that locoregional therapy such as surgery and radiation may be of benefit to select patients with CUP. A retrospective study by Löffler and colleagues reported that patients with a limited local involvement who received radical surgery had a median overall survival of 52.7 months compared with those who received radiation (median overall survival, 19.4 months) and those who received chemotherapy alone (median overall survival, 16 months). ${ }^{13}$ A case of a metastatic undifferentiated CUP also reported a long-term ( $>5$ years), disease-free survivor after pancreaticoduodenectomy and systemic adjuvant chemotherapy. ${ }^{14}$

Our case further demonstrates that a multidisciplinary approach to CUP may lead to excellent clinical outcomes. Chemotherapy followed by chemoradiation in our patient increased local tumor control and survival. Our patient's 44-month survival was superior to the historic 6.5- to 13-month median survival in CUP patients treated with chemotherapy alone. Consolidation chemoradiation treatment may therefore be a viable and more effective therapy in the treatment of adenocarcinoma of unknown primary, in which anatomical disease concentration is amenable to radiotherapy following control with systemic chemotherapy. Nevertheless, it is difficult to draw conclusions from select cases. Another case of mediastinal adenocarcinoma, favoring a colorectal primary but with no evidence of a 
primary lesion on endoscopy, had a poorer outcome than did our patient, with the cancer recurring 6 months after completion of chemotherapy, surgical excision, and adjuvant radiotherapy. ${ }^{15}$

\section{References}

1. Pavlidis N, Khaled H, Gaafar R. A mini review on cancer of unknown primary site: a clinical puzzle for the oncologists. J Adv Res. 2015;6(3):375-382.

2. Pavlidis N, Briasoulis E, Hainsworth J, Greco FA. Diagnostic and therapeutic management of cancer of an unknown primary. Eur J Cancer. 2003;39(14):1990-2005.

3. Oien KA. Pathologic evaluation of unknown primary cancer. Semin Oncol. 2009;36(1):8-37.

4. Louhimo J, Alfthan H, Stenman UH, Hagland C. Serum HCG beta and CA 72-4 are stronger prognostic factors than CEA, CA 19-9 and CA 242 in pancreatic cancer. Oncology. 2004;66(2):126-131.

5. Conroy T, Desseigne F, Ychou M, et al. FOLFIRINOX versus gemcitabine for metastatic pancreatic cancer. N Engl J Med. 2011;364(19):1817-1825.

6. Pavlidis N, Pentheroudakis G. Cancer of unknown primary site. Lancet. 2012;379:1428-1435.

7. Bochtler T, Löffler H, Krämer A. Diagnosis and management of metastatic neoplasms with unknown primary. Semin Diagn Pathol. 2017;35(3):199-206.

8. Amela EY, Lauridant-Philippin G, Cousin S, Ryckewaert T, Adenis A, Penel N. Management of 'unfavourable' carcinoma of unknown primary site: synthesis of recent literature. Crit Rev Oncol Hematol. 2012;84(2):213-223

9. Culine S, Lortholary A, Voigt J-J, et al. Cisplatin in combination with either gemcitabine or irinotecan in carcinomas of unknown primary site: results of a randomized phase II study—-trial for the
Adenocarcinomas of unknown primary cases should involve management by a multidisciplinary team. Clinical trials incorporating locoregional therapies for CUP in addition to systemic therapy are warranted.

French study group on carcinomas of unknown primary (GEFCAPI 01). J Clin Oncol. 2003;21(18):3479-3482.

10. Hainsworth JD, Spigel DR, Thompson DS, et al. Paclitaxel/carboplatin plus bevacizumab/erlotinib in the first-line treatment of patients with carcinoma of unknown primary site. Oncologist. 2009;14(12):1189-1197.

11. Kubisch CH, Beigel F, Ihrler S, Goke B, Reiser MF, Hoffmann RT. Oesophageal ulceration after selective internal radiation therapy in a patient with carcinoma of unknown primary. Z Gastroenterol. 2010;48(5):546-550.

12. Shiota $Y$, Imai S, Sasaki N, et al. A case of mediastinal lymph node carcinoma of unknown primary site treated with docetaxel and cisplatin with concurrent thoracic radiation therapy. Acta Med Okayama. 2011;65(6):407-411.

13. Löffler H, Puthenparambil J, Hielscher T, Neben K, Krämer A. Patients with cancer of unknown primary: a retrospective analysis of 223 patients with adenocarcinoma or undifferentiated carcinoma. Dtsch Arztebl Int. 111(27-28):481-487.

14. Nakagawa Y, Todoroki T, Morishita Y, et al. A long-term survivor after pancreaticoduodenectomy for metastatic undifferentiated carcinoma of an unknown primary. Hepatogastroenterology. 2008;55(86-87):1557-1561.

15. Rodríguez-López JL, Toro-Bahamonde AM, Santiago-Méndez RJ, González-Cancel IF, Vélez-Cortés HA. An unusual case of colorectal adenocarcinoma presenting as an anterior mediastinal mass. Clin Colorectal Cancer. 2018;17(1):e115-e119. 